\title{
CIDOFOVIR AND INTRAVENOUS IMMUNOGLOBULIN FOR TREATMENT OF BK POlYOMAVIRUS NePHROPATHY
}

Anita Mehrotra, MD, Rajani Dinavahi, MD, and George C. Francos, MD

\section{Abstract}

BK polyomavirus nephropathy (BKN) is an important cause of renal allograft dysfunction and loss. The mainstay of therapy is reduced immunosuppression, but definitive protocols for the diagnosis and management remain to be defined. We report here a retrospective analysis of 6 patients with BKN treated with cidofovir and intravenous immunoglobulin(IVIG). The patients were followed for 6 months from the initiation of therapy. All patients (3 cadaveric/3 live donors) underwent renal biopsy after serum BK viral PCR (SBKPCR) was obtained in response to a rise in creatinine concentration. The mean time from transplantation to diagnosis was 21.7 months $(5,14,15,24,35,35$ months). One patient had positive SBKPCR without biopsy evidence of BKN. In all patients immunosuppression was reduced, and therapy with cidofovir $(0.25-0.5 \mathrm{mg} / \mathrm{kg})$ and IVIG (25 grams) was instituted weekly or bi-weekly. Mycophenolate mofetil was discontinued, and the tacrolimus therapeutic trough was decreased to $2-5 \mathrm{ng} / \mathrm{mL}$ in $5 / 6$ patients. Therapy was continued for 6 months or until SBKPCR was undetectable. The mean nadir creatinine value after transplantation was $1.3 \mathrm{mg} / \mathrm{dL}$ $(1,1.1,1.1,1.2,1.3,1.8)$. The mean creatinine value at time of biopsy was $2.3 \mathrm{mg} / \mathrm{dL}(1.2,1.4,1.7,2.8,3,3.6)$, and the mean
SBKPCR value at diagnosis was 870,266 copies/mL $(1.86,23$, $33,38,56,370 \times 104$ copies $/ \mathrm{mL})$. The average number of cidofovir doses was 8.3 (4, 6,6, 10, 12, 12 doses) with concomitant IVIG. The mean SBKPCR at end of 6 months was 44,283 copies/dL (1.1, 1.8, 2.4, 5.4, 10, $245 \times 103$ copies/mL). Five of 6 patients experienced a decrease in SBKPCR. The mean creatinine value at the end of 6 months was $3.0 \mathrm{mg} / \mathrm{dL}(1.5,2$, $2.9,3.3,3.7,4.4)$. After nine months from initiation of therapy, the one patient with positive SBKPCR and biopsy negative for BKN returned to a nadir creatinine $(1.2 \mathrm{mg} . / \mathrm{dL})$ and undetectable SBKPCR. Baseline renal function (the nadir creatinine prior to the diagnosis of BK infection), was not recovered in any patient at the end of six months. These data suggest that the reduction of immunosuppression and addition of cidofovir and intravenous immunoglobulin can arrest further progression of BKN. Allograft dysfunction, however, was not reversed and may reflect fibrosis secondary to the injury sustained as a result of BKN. The one patient diagnosed by SBKPCR prior to the development of BKN had the most favorable outcome. Serologic screening prior to development of allograft dysfunction may be warranted, but the timing and treatment strategies need to be confirmed by larger studies.
Yeongbokgung Palace Seoul, Korea

Photo courtesy of Eric Choi, $M D$

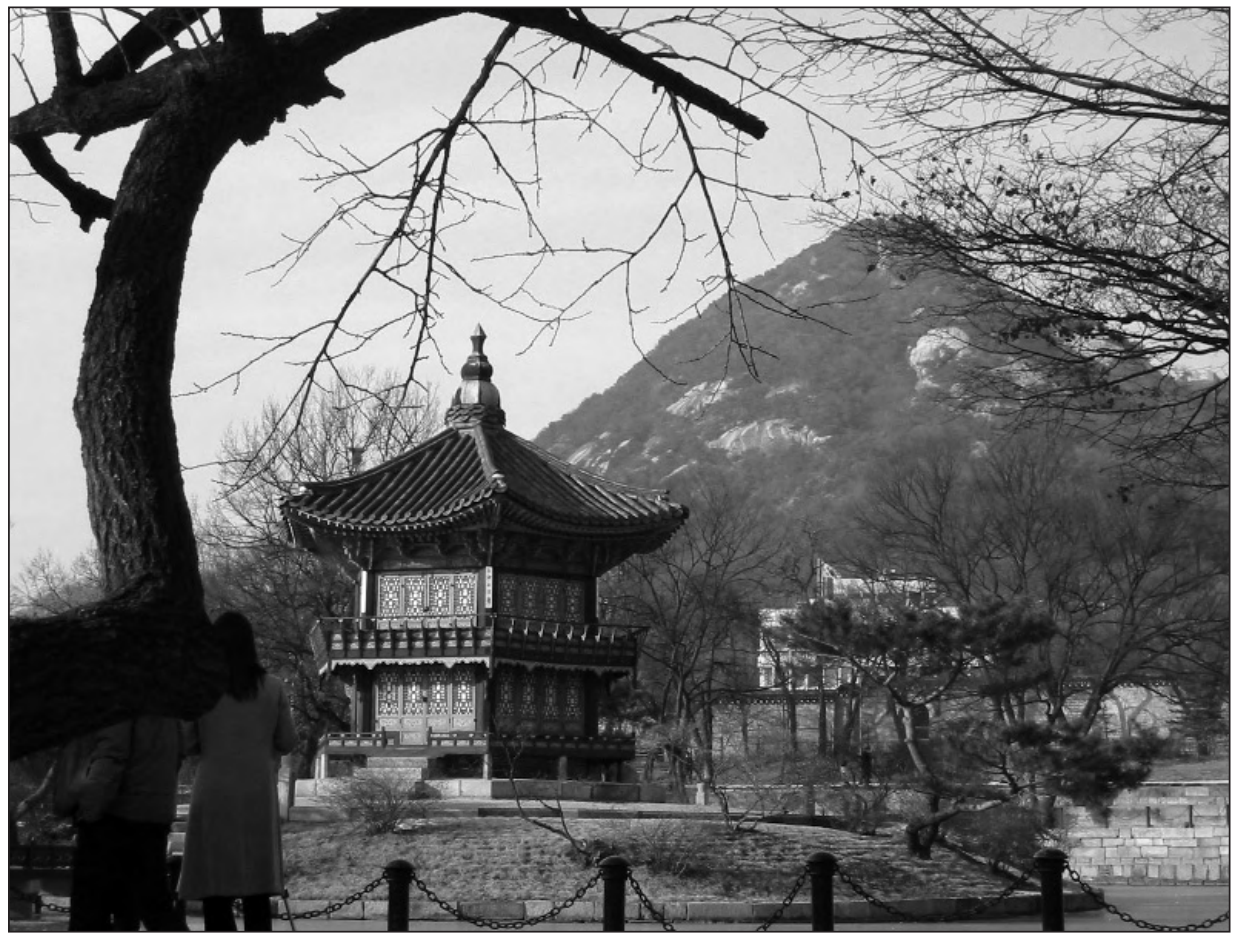

